\title{
When is the concept of generalized transport costs useless? The effects of the change in the value of time
}

\author{
T. Kono ${ }^{1}$, H. Morisugi ${ }^{2} \&$ A. Kishi ${ }^{3}$ \\ ${ }^{I}$ Graduate School of Engineering, Tohoku University, Japan \\ ${ }^{2}$ Graduate School of Economics, Tohoku University, Japan \\ ${ }^{3}$ Department of Administration and Informatics, University of Shizuoka, \\ Japan
}

\begin{abstract}
Under an exogenous working time assumption, the value of time cannot be constant: it is affected by travel time, travel fees and other exogenous factors. Our numerical simulations, assuming a nested C.E.S. form of the utility function, show that the change in value of time tends to be sensitive to travel time and slightly sensitive to travel fees. Based on the change in value of time, the influences of changes in travel time and travel fees on the number of visits can differ greatly even if those changes are equivalent in terms of generalized transportation costs.
\end{abstract}

Keywords: value of time, private trip, trip demand, generalized travel cost.

\section{Introduction}

The value of time is one of the most important variables for both trip demand forecasting and project appraisal. Almost all present practices in both trip demand forecasting and project appraisal assume the constancy of value of time, which often adopts a wage rate, as shown by Hayashi and Morisugi [7]. On the other hand, some papers, e.g., DeSerpa [6], Bruzelius [3] and Jara-Diaz [10], point out that value of time can differ from the wage rate for several reasons including fixed work hours, disutility from work, or uncertainty in travel time. Among those reasons, this paper specifically addresses fixed work hours.

As is well known, under the assumption that individuals can freely choose working-time and that working is utility-neutral, the value of time is equal to the 
wage. The widely used "wage rate approach", in which the value of time is the wage rate, is based on this consequence.

Although that assumption might be applicable to lawyers and individual consultants, that assumption is not realistic in many situations in not only the short run, but also the long run. For example, most office workers are strongly dependent on other persons' works. Hence, they cannot continuously change their working time to suit their preference. They could choose working time discretely at best (e.g. they quit the job or not). In the case of persons who do not work for business, e.g., full time homemakers, students and pensioners, they can be regarded as not choosing their working times with given exogenous income. In other words, their working times are fixed to be zero but they are given money from their partner, parents, governments or others.

Under an exogenous working time assumption, the value of time for private trips cannot be constant or equal to the wage rate. Instead, it is affected by various factors including travel time, travel fees, and others. Project appraisal accordingly needs to take their influence on value of time into account. Moreover, the trip demand should be forecasted with the endogenous value of time simultaneously.

Some empirical studies have specifically addressed the non-constancy of value of time, e.g., Calfee and Winston [4] Brownstone et al. [2], and others. Their estimates are based on discrete choice analysis, e.g., Small and Rosen [12] and Anderson et al. [1]. Among them, Hensher [8] attempts estimation of the (time) value function with SP data, i.e., he estimates the change in value of time associated with the change in travel time. Lam and Small [11] estimates the variation in value of time depending on trip distance and others with RP data. Theoretical studies by DeSerpa [6], and deDonnea [5] also have already pointed out that value of time changes depending on the economic environment of individuals under exogenous working time assumption.

This study simulates how much the value of time changes and analyzes the interaction between the number of trips and the endogenous value of time quantitatively. Specifically, we set a nested C.E.S. (i.e., Constant Elasticity of Substitution) form of the utility function to numerically simulate the travel time and travel fee elasticities of value of time and trip demand. Simulation results show that, for shopping trips, the value of time tends to change relatively much for the change in the travel time whereas the value of time changes moderately for the change in the travel fee. Accordingly, the simulation shows that the elasticities of trip demand are also very different with regard to that between travel fee and travel time.

This great difference means that the conventional trip demand estimation method, e.g., a four-step method, which adopts the concept of generalized transportation costs, could be inadequate for trip demand forecasting. Conventional trip demand estimation method expresses the trip demand as a function of the generalized travel cost comprising the travel fee and the constant value of time multiplied by the travel time. Thereby, the change in trip demand that is attributable to the change in travel time is the same as the change in trip demand caused by the change in the travel fee equal to "fixed value of time 
multiplied by the change in travel time" in the conventional method because the travel time and the travel fee influence the demand equivalently in terms of the generalized travel cost. On the other hand, our simulation shows that these changes in trip demand differ greatly under the condition of endogenous working time assumption.

The reason for this difference can be explained as follows. Under the exogenous working time assumption, the change in travel time or travel fee influences the endogenous value of time in the opposite sign, i.e., the increase in travel time increases the value of time, whereas the increase in travel fee decreases the value of time. The value of time affects trip demand directly. Therefore, the opposite results directly generate the difference in the effect of the changes of between travel time and travel fees on trip demand. Moreover, the change in value of time affects not only the demand for goods requiring trips for purchase, but also the other goods, e.g., composite goods and leisure time. Those indirect effects also affect the trip demand. Hence, even if the change in value of time itself is small because of changes in travel times or travel fees, the change in trip demand can be vast through direct and indirect effects.

Section 2 shows the model and the set of parameters for numerical simulations. Section 3 shows the numerical simulations to obtain the travel time and travel fee elasticities of value of time and trip demand. Section 4 concludes this study.

\section{Numerical simulations on changes in the value of time}

This section constructs the model treating the value of time endogenously. We calculate the change in the value of time caused by the change in travel time and travel fees for the case of shopping trips.

\subsection{Formation of an individual's behavioral model}

We assume that there are two shopping places (one in the urban area and another in the suburban area) and each consumer chooses the number of visits to each place, the amount of the composite good, and the amount of leisure time. The amount of composite goods per visit at each place and the shopping time per visit at each place are fixed for simplicity. We then specify consumers' direct utility function as a nested C.E.S. form (i.e., Constant Elasticity of Substitution form). The consumers' utility maximization is expressed as

$$
\begin{gathered}
V(\cdot)=\max _{x_{k i}, Z_{z}, Z_{h}}\left[\alpha_{s}\left(\sum_{i=1}^{2} \beta_{i} x_{k i}^{\eta_{2}}\right)^{\frac{\eta_{1}}{\eta_{2}}}+\alpha_{z} Z_{z}^{\eta_{1}}+\alpha_{h} Z_{h}^{\eta_{1}}\right]^{\frac{1}{\eta_{1}}}, \\
\text { s.t. } \quad Z_{z}+\sum_{i=1}^{n}\left(P_{i}+x_{s i}\right) x_{k i}=I-P_{1} x_{w}, \quad \text { and } \\
Z_{h}+\sum_{i=1}^{n}\left(\tau_{t i}+t_{t i}\right) x_{k i}=T-x_{w} \tau_{t 1},
\end{gathered}
$$


where $Z_{z}$ is the amount of the composite good; $Z_{h}$ is the amount of leisure time; $x_{k i}$ is the number of visits to shopping place $i(i=1,2)(1$ is located in the urban area and 2 in the suburban area); $x_{s i}$ is the amount of consumption at place $i$ per visit; $x_{w}$ is the number of commuting trips; $t_{t i}$ is the staying hours at place $i ; I$ is total income; $T$ is total available time excluding working hours; $P_{i}$ is the travel fee for a round trip; $\tau_{t i}$ is the travel time for a round trip; $\alpha_{h}, \alpha_{s}, \alpha_{z}, \beta_{i}, \eta_{1}$

and $\eta_{2}$ are parameters $\left(\alpha_{s}+\alpha_{h}+\alpha_{z}=1, \sum_{i=1}^{2} \beta_{i}=1\right) ; V(\cdot)$ is the indirect utility function.

It is assumed that the individual commutes to the same place as shopping place 1: the city area. Furthermore, all goods prices are fixed at unity for simplicity. Exogenous variables for consumers are: total income $I$, total available time excluding working hours $T$, the staying hours at place $i t_{t i}$, the travel fee $P_{i}$, the amount of consumption at place $i$ per one visit $x_{s i}$, the number of commuting trips $x_{w}$, and travel time to place $i \tau_{t i}$. From eqns. (1), (2), and (3), endogenous variables are leisure time $Z_{h}$, composite good $Z_{z}$, the number of visits to shopping place $i x_{k i}$, and value of time.

Lagrangian function $S$ expressing eqns. (1)-(3) is given as

$$
\begin{aligned}
& S=\left[\alpha_{s}\left(\sum_{i=1}^{2} \beta_{i} x_{k i}^{\eta_{2}}\right)^{\frac{\eta_{1}}{\eta_{2}}}+\alpha_{z} Z_{z}^{\eta_{1}}+\alpha_{h} Z_{h}^{\eta_{1}}\right]^{\frac{1}{\eta_{1}}} \\
& +\lambda\left[I-P_{1} x_{w}-Z_{z}-\sum_{i=1}^{2}\left(P_{i}+x_{s i}\right) x_{k i}\right]+\mu\left[T-x_{w} \tau_{t 1}-Z_{h}-\sum_{i=1}^{2}\left(\tau_{t i}+t_{t i}\right) x_{k i}\right]
\end{aligned}
$$

where $\lambda$ and $\mu$ are Lagrangian multipliers associated with income and time constraints. As mentioned before, value of time, VoT, is expressed as

$$
\operatorname{VoT}=\frac{\mu^{*}}{\lambda^{*}} .
$$

Solving the utility maximization problem (1)-(3) numerically, we can obtain the value of time, $V o T$, from (5).

\subsection{Setting of parameters}

This study carries out numerical simulations, setting the values of the exogenous variables as shown in table 1 . These fundamental variables are set based on the following real and hypothetical data set. The total income per month is taken from data of disposable income of Sendai city in Japan in 1998. The total available time, which is defined as the rest of the day excluding working time, sleeping time and housekeeping time, is determined from questionnaires taken in 
the city of Sendai in 2000. The total available time is estimated as 21,300 minutes per month (11.6 hours per day on the average, both for weekdays and weekend). Other variables such as travel time and travel fees are set as those of a consumer living in a suburban area in Sendai.

Table 1: $\quad$ Fundamental exogenous variables (per month).

\begin{tabular}{|c|c|c|c|c|c||c|c|}
\hline & $\begin{array}{c}x_{s i} \\
(\text { yen})\end{array}$ & $\begin{array}{c}x_{w} \\
\text { (trips } \\
\text { ) }\end{array}$ & $\begin{array}{c}t_{t i} \\
\text { (minutes) }\end{array}$ & $\begin{array}{c}P_{i} \\
(\text { yen })\end{array}$ & $\begin{array}{c}\tau_{i i} \\
\text { (minutes) }\end{array}$ & $\begin{array}{c}I \\
(\text { yen })\end{array}$ & $\begin{array}{c}T \\
(\text { minute } \\
\text { s) }\end{array}$ \\
\hline $\begin{array}{c}\text { City area } \\
(i=1)\end{array}$ & 10000 & 20 & 60 & 1000 & 90 & 12969 & 21300 \\
\hline Suburb $(i=2)$ & 3000 & - & 60 & 300 & 30 & 4 & \\
\hline
\end{tabular}

Next, we set parameters of the utility function. This paper assumes these parameters shown in table 2 . The elasticity of substitution between place 1 and place $2, \eta_{2}$, is set in two ways. The first is a strong elasticity of substitution between place 1 and place 2 , that is, $\eta_{2}=0.9$. The other case is a weak elasticity of substitution between place 1 and place 2 , that is, $\eta_{2}=0.3$.

Table 2: $\quad$ Parameter set of the behavioral model.

\begin{tabular}{|c|c|c|c|c|c|c|}
\hline$\alpha_{s}$ & $\alpha_{z}$ & $\alpha_{h}$ & $\beta_{1}$ & $\beta_{2}$ & $\eta_{1}$ & $\eta_{2}$ \\
\hline 0.8 & 0.06 & 0.194 & 0.6 & 0.4 & 0.5 & 0.9 \\
\hline
\end{tabular}

Setting the exogenous variables in table 2 and the assumed parameters in table 2, we can estimate the value of time and other endogenous variables by solving the utility maximization problem eqns. (1)-(3) numerically. Table 3 shows the result of estimation.

Table 3: $\quad$ Results of estimation (per month).

\begin{tabular}{|c|c|c|c|c||c|}
\hline & $x_{k 1}$ (trips) & $x_{k 2}$ (trips) & $Z_{z}$ (yen) & $Z_{h}$ (minutes) & $\begin{array}{c}V o T \text { (yen/ } \\
\text { minute) }\end{array}$ \\
\hline$\eta_{2}=0.9$ & 5.97 & 8.79 & 33039 & 19434 & 42.16 \\
\hline$\eta_{2}=0.3$ & 6.81 & 7.25 & 28897 & 19447 & 39.41 \\
\hline
\end{tabular}

\section{Comparative statics on trip demand}

This section carries out numerical simulations on the change in the value of time concerning the change in travel time and travel fee. The travel time to place 
$1, \tau_{t 1}$, is changed from 30 minutes to 150 minutes. The travel fee to place $1, P_{1}$, is changed from 157 yen to 1843 yen for $\eta_{2}=0.9$, and from 212 yen to 1788 yen for $\eta_{2}=0.3$. Table 4 and Table 5 show the calculation result.

Table 4: Comparative statics with regard to travel time.

\begin{tabular}{|c|c|c|c|c|c|c|c|}
\hline \multicolumn{8}{|c|}{$\eta_{2}=0.9$} \\
\hline$\tau_{t 1}$ & 30 & 50 & 70 & 90 & 110 & 130 & 150 \\
\hline$Z_{z}$ & 33098 & 33035 & 33036 & 33039 & 33042 & 33044 & 33183 \\
\hline$Z_{h}$ & 21354 & 20726 & 20087 & 19434 & 18771 & 18112 & 17500 \\
\hline$x_{k 1}$ & 7.68 & 7.30 & 6.75 & 5.97 & 4.87 & 3.40 & 1.75 \\
\hline$x_{k 2}$ & 3.05 & 4.34 & 6.17 & 8.79 & 12.46 & 17.35 & 22.81 \\
\hline VoT & 40.25 & 40.82 & 41.47 & 42.16 & 42.90 & 43.67 & 44.52 \\
\hline \multicolumn{8}{|c|}{$\eta_{2}=0.3$} \\
\hline$\tau_{t 1}$ & 30 & 50 & 70 & 90 & 110 & 130 & 150 \\
\hline$Z_{z}$ & 28801 & 28803 & 28893 & 28897 & 28901 & 28906 & 28911 \\
\hline$Z_{h}$ & 21146 & 20577 & 20027 & 19447 & 18886 & 18330 & 17780 \\
\hline$x_{k l}$ & 7.25 & 7.13 & 7.07 & 6.81 & 6.62 & 6.41 & 6.16 \\
\hline$x_{k 2}$ & 5.80 & 6.21 & 6.37 & 7.25 & 7.87 & 8.58 & 9.40 \\
\hline VoT & 37.73 & 38.25 & 38.84 & 39.41 & 40.00 & 40.60 & 41.23 \\
\hline
\end{tabular}

Table 5: Comparative statics with regard to travel fees.

\begin{tabular}{|c|c|c|c|c|c|c|c|}
\hline \multicolumn{8}{|c|}{$\eta_{2}=0.9$} \\
\hline$P_{1}$ & 157 & 500 & 750 & 1000 & 1250 & 1500 & 1843 \\
\hline$Z_{z}$ & 33039 & 33039 & 33039 & 33039 & 33039 & 33039 & 33039 \\
\hline$Z_{h}$ & 19433 & 19434 & 19434 & 19434 & 19434 & 19434 & 19434 \\
\hline$x_{k 1}$ & 10.67 & 8.56 & 7.20 & 5.97 & 4.84 & 3.80 & 2.52 \\
\hline$x_{k 2}$ & 0.96 & 4.47 & 6.73 & 8.79 & 10.67 & 12.40 & 14.54 \\
\hline VoT & 42.1591 & 42.1590 & 42.1589 & 42.1589 & 42.1588 & 42.1588 & 42.1587 \\
\hline \multicolumn{8}{|c|}{$\eta_{2}=0.3$} \\
\hline$P_{1}$ & 212 & 500 & 750 & 1000 & 1250 & 1500 & 1788 \\
\hline$Z_{z}$ & 28951 & 28935 & 28913 & 28897 & 28897 & 28897 & 28897 \\
\hline$Z_{h}$ & 19067 & 19235 & 19342 & 19447 & 19447 & 19447 & 19447 \\
\hline$x_{k l}$ & 8.33 & 7.93 & 7.35 & 6.81 & 5.64 & 4.57 & 3.45 \\
\hline$x_{k 2}$ & 8.92 & 7.73 & 7.50 & 7.25 & 9.19 & 10.97 & 12.84 \\
\hline VoT & 39.8418 & 39.6571 & 39.5313 & 39.4143 & 39.4142 & 39.4142 & 39.4142 \\
\hline
\end{tabular}




\subsection{The change in the value of time}

Table 4 shows that value of time changes by approximately $10 \%$ according to the change in travel time, whereas Table 5 shows a very small change in the value of time with respect to change in travel fee. In terms of elasticity, the elasticity of the value of time with regard to travel time from 90 minutes to 30 minutes is 0.068 for $\eta_{2}=0.9$ and 0.064 for $\eta_{2}=0.3$. On the other hand, the elasticity of the value of time with regard to the travel fee from 1000 yen to 500 yen is almost zero for $\eta_{2}=0.9$ and 0.012 for $\eta_{2}=0.3$. The elasticity of the value of time with regard to travel time is very large compared to that with regard to travel fee. The difference of $\eta_{2}$, the elasticity of substitution between place 1 and place 2 , obviously affects the change in the number of visits, but has only a very small effect on the range of the change in the value of time.

\subsection{The change in trip demand}

The changes in trip demand with regard to changes in travel cost and the equivalent travel time should be the same if the trip demand is determined by the generalized transportation cost composed of travel time times the value of time and travel fee. However, the simulation results engender the conclusion that influences on the number of visits of travel time and travel fees can differ relatively much even if the change in the travel fee were equivalent to travel time in terms of generalized transportation cost, as follows.

The change of 20 minutes in travel time to place 1 from 90 minutes is equivalent to 843 yen $(=42.16 \mathrm{yen} / \mathrm{min} \times 20 \mathrm{~min})$ in the case of $\eta_{2}=0.9$ and 788 yen $(=39.41 \mathrm{yen} / \mathrm{min} \times 20 \mathrm{~min})$ in the case of $\eta_{2}=0.3$ if the value of time were assumed to be constant at 90 minutes travel time. However, the number of visits in the cases $\left(P_{1}=1000, \tau_{1}=70\right)$ and $\left(P_{1}=157, \tau_{1}=90\right)$ for $\eta_{2}=0.9$ or ( $\left.P_{1}=212, \tau_{1}=90\right) \eta_{2}=0.3$ are quite different. For $\eta_{2}=0.9$, the number of visits to place 1 in the case of $\left(P_{1}=1000, \tau_{1}=70\right)$ is 6.75 , whereas the number of visits to place 1 in the case of $\left(P_{1}=157, \tau_{1}=90\right)$ is 10.67 . For $\eta_{2}=0.3$, the number of visits to place 1 in the case of $\left(P_{1}=1000, \tau_{1}=70\right)$ is 7.07 , whereas the number of times to visit place 1 in the case of $\left(P_{1}=212, \tau_{1}=90\right)$ is 8.33 . The same things can be said for cases of $\left(P_{1}=1000, \tau_{1}=110\right)$ and $\left(P_{1}=1843, \tau_{1}=90\right)$ for $\eta_{2}=0.9$ or $\left(P_{1}=1788, \tau_{1}=90\right)$ for $\eta_{2}=0.3$.

\subsection{Implications of the simulation results}

The simulation results indicate that the conventional estimation method is entirely wrong under the condition of fixed working time. In the conventional demand estimation method, the travel demand depends on the generalized transportation cost composed of travel fee and the value of time times the travel time. That means that the change in demand that is attributable to the change in 
the travel time is the same as the change in demand caused by the change in travel fee equal to "value of time times the change in travel time" in the conventional method because the travel time and the travel fee influence the demand equivalently through the generalized travel cost. Our simulation shows that these changes in trip demand are differ markedly under the condition of fixed working time. This fact might explain the reported differences between the estimated demand by the conventional method and the actual traffic demand with the endogenous value of time.

The reason for different changes in demand that occur through the changes between the travel time and the equivalent travel fee are quite different can be explained as follows. The change in travel time and travel fee makes the value of time change in the opposite way: the increase in travel time increases the value of time, whereas the increase in travel fees decreases the value of time. One can confirm the opposite sign in changes in value of time in table 4 and table 5 as follows. Table 4 shows that the value of time changes from 42.16 to 42.90 with regard to the change in travel time from 90 minutes to 110 minutes at $\eta_{2}=0.9$, whereas Table 5 shows that the value of time changes from 42.1589 to 42.1587 with regard to the change in travel fee from 1,000 yen to 1,843 yen at $\eta_{2}=0.9$.

The opposite results generate the difference in the effect of the changes of between travel time and travel fee on trip demand because the value of time directly affects trip demand. Aside from that fact, the change in value of time affects not only the demand of goods requiring trips for purchase, but also that of other goods, e.g., composite goods and leisure time. One can see this fact regarding leisure time in Table 4 and Table 5 as follows. Table 4 shows that the leisure time changes from 19,434 to 18,771 with regard to the change in travel time from 90 minutes to 110 minutes at $\eta_{2}=0.9$, whereas Table 5 shows that the leisure time changes from 19,434 to 19,434, i.e., the changes are negligible in this case, with regard to the change in travel fee from 1000 yen to 1843 yen at $\eta_{2}=0.9$. The other goods, e.g., the composite goods and leisure time, affect the number of trips through income or time constraints. Hence, even changes in travel time or travel fees induce only small changes in the value of time: the change in demand can be vast because of changes in travel time or travel fees.

\section{Conclusions}

Contrary to common assumptions of conventional economics, such as full choice of work time such that the value of time is equal to the wage, this study assumes that working time is an exogenous variable. Under this assumption, the value of time depends on the economic environment according to commodity prices, travel time, and other factors. This paper first clarifies how the value of time changes depending on the economic environment, assuming a C.E.S. form of the utility function.

Based on our simulation results, the value of time tends to be sensitive to travel time whereas there is no sensitivity for the travel fee. The elasticity of the value of time with regard to travel time is very large compared to that of the 
travel fee. Furthermore, our simulation shows that the change in the number of visits through changes in travel time and travel fees can differ greatly even if the change in the travel fee is equivalent to travel time in terms of generalized transportation cost. This result is important for trip demand estimation. In the conventional demand estimation method, the travel demand depends on the generalized transportation cost, which comprises travel fee and the "value of time times the travel time". That means that changes in demand with regard to changes in "travel time multiplied by the value of time" is the same as the change in trip demand with regard to travel fees in the conventional method if the changes in "travel time times value of time" and travel fees are the same. Our simulation shows that those changes in demand are totally different under the condition of fixed working time.

The trip demand is determined simultaneously with the endogenous value of time. In that sense, the change in value of time should be taken into account when forecasting trip demand. Results of our simulations show that the value of time can differ up to $10 \%$ depending on reasonable changes in travel time. Results suggest that the forecast method should take the change in value of time into consideration. If the value of time is not considered, the consequently forecasted trip demand would reflect neither the value of time nor the real trip demand.

\section{References}

[1] Anderson S, de Palma A, J-F. Thisse, Discrete choice theory of product differentiation, MIT Press: Cambridge, MA, 1992.

[2] Brownstone D, Kazime C, Ghosh A, Golob TF, and van Amelsfort D, Drivers' willingness-to-pay to reduce travel time: evidence from the San Diego I-15 congestion pricing project, Transportation Research A 37:373387, 2003.

[3] Bruzelius N, The value of travel time, Croom Helm: London, 1978.

[4] Calfee J, Winston C, The value of automobile travel time: Implications for congestion policy, Journal of Public Economics 69:83-102, 1998,

[5] deDonnea FX, Consumer behaviour, transport mode choice and value of time: some micro-economic models, Regional and urban economics 1:355382,1972 .

[6] DeSerpa AC, A theory of the economics of time, The Economic Journal 81:828-846, 1971,

[7] Hayashi Y, Morisugi H, 2000, International Comparison of Background Concept and Methodology of Transportation Project Appraisal, Transport Policy 7, No.1: 73-88.

[8] Hensher DA, Review of Studies Leading to Existing Values of Travel Time. Transportation Research Record, 587:30-41,1976.

[9] Hensher DA, Behavioral Value of Travel Time Savings in Personal and Commercial. In: Greene DL, Jones DW, Delucchi MA (eds) The Full Costs and Benefits of Transportation, Springer-Verlag, 1997. 
[10] Jara-Diaz SR, Allocation and valuation of travel-time savings, In: Hensher DA, Button KJ (eds) Handbook of Transport Modelling 1. Oxford Elsevier Science, 303-318, 2000.

[11] Lam TC, Small KA, The Value of Time and Reliability: Measurement from a Value Pricing Experiment, Transportation Research Part E 37:231-251, 2001 ,

[12] Small KA, Rosen HS, Applied welfare economics with discrete choice models. Econometrica 49:105-130, 1992. 